\title{
The effects of Helicobacter pylori infection on pregnancy-related diseases and fetal development in diabetes in pregnancy
}

\author{
Jun Li ${ }^{1}$, Mengdi Fan ${ }^{1}$, Fei Ma ${ }^{2}$, Suhe Zhang ${ }^{1}$, Qingju Li ${ }^{1}$ \\ ${ }^{1}$ Endocrinology Department, The Second Affiliated Hospital of Zhengzhou University, Zhengzhou, China; ${ }^{2}$ General Surgery Department, The \\ Affiliated Tumor Hospital of Zhengzhou University, Zhengzhou, China \\ Contributions: (I) Conception and design: J Li, Q Li; (II) Administrative support: S Zhang, Q Li; (III) Provision of study materials or patients: J Li, M \\ Fan, F Ma; (IV) Collection and assembly of data: J Li, M Fan; (V) Data analysis and interpretation: J Li, M Fan, F Ma; (VI) Manuscript writing: All \\ authors; (VII) Final approval of manuscript: All authors. \\ Correspondence to: Qingju Li. Endocrinology Department, The Second Affiliated Hospital of Zhengzhou University, No. 2 Jingba Road, Jinshui \\ District, Zhengzhou 450014, China. Email: liqingju1133@163.com.
}

Background: This study aimed to determine the association of Helicobacter pylori (H. pylori) infection with pregnancy-related diseases and fetal development in women with diabetes in pregnancy (DIP).

Methods: All the participants were recruited before 16 weeks of gestation. According to their medical history and the results of a $75-\mathrm{g}$ oral glucose tolerance test at the $24^{\text {th }}$ week of pregnancy, the participants were divided into a normal control group (NC group), a gestational diabetes mellitus group (GDM group), and a pre-pregnancy diabetes mellitus group (PGDM group). According to the results of an H. pylori serum antibody detection test, each group was further divided into two subgroups: an $H$. pylori positive subgroup (HP+ subgroup) and an $H$. pylori negative group (HP-subgroup). The incidences of pregnancy-related diseases, the fetal developmental status, and the newborn status were compared among the groups.

Results: This study recruited 356 pregnant women. The infection rates of type I H. pylori were significantly higher in the GDM group and the PGDM group than in the NC group $\left(\chi^{2}=6.949, \mathrm{P}=0.031\right)$. With the exception of the NC-HP+ subgroup, there were higher incidences of pregnancy-related diseases in the $H P+$ subgroups than in the $H P$ - subgroups $(\mathrm{P}<0.05)$. Furthermore, the incidences of pregnancyinduced hypertension $(\mathrm{PIH})$, preeclampsia, and premature delivery were significantly higher in the GDM$H P+$ subgroup and the PGDM-HP+ subgroup than in the $\mathrm{NC}-H P+\operatorname{subgroup}(\mathrm{P}<0.05)$. At the end of pregnancy, all $3 H P$ - subgroups showed better fetal development than the $H P+$ subgroups $(\mathrm{P}<0.05)$, and the NC-HP+ subgroup showed better fetal development than the GDM- $H P+$ and PGDM-HP+ subgroups $(\mathrm{P}<0.05)$. Meanwhile, the PGDM-HP+ subgroup showed poor fetal development, even in the $2^{\text {nd }}$ trimester of pregnancy.

Conclusions: $H$. pylori infection is extremely common in DIP. For women with DIP, infection with $H$. pylori can increase the risks of pregnancy-related diseases and poor fetal development. $H$. pylori screening and eradication therapy before pregnancy may aid in preventing pregnancy-related diseases and improve fetal development.

Keywords: Helicobacter pylori (H. pylori); diabetes in pregnancy (DIP); pregnancy-related diseases; fetal development

Submitted Feb 23, 2021. Accepted for publication Apr 15, 2021.

doi: 10.21037/atm-21-1209

View this article at: http://dx.doi.org/10.21037/atm-21-1209 


\section{Introduction}

Preeclampsia (PE), pregnancy-induced hypertension (PIH), hyperemesis gravidarum (HG), and anemia of pregnancy are serious disorders occurring during pregnancy, which have adverse effects on both maternal and fetal safety. Poor fetal development, indicated by a low biparietal diameter and small head circumference as well as a low birth weight and short body length at birth, are associated with neonatal death. However, the etiology and the pathogenesis of pregnancy-related diseases and poor fetal development are still poorly understood. Diabetes mellitus (DM) and infection may play roles in the etiology and pathogenesis of pregnancy-related complications and poor development of a fetus.

Helicobacter pylori $(H$. pylori) is a gram-negative bacterium with a specific tropism for the gastric mucosa (1). It has been found to be the main cause of chronic gastric diseases (2). Almost half of the world's population is infected with $H$. pylori infection, with the infection rates being higher in developing countries $(3,4)$. According to 2015 estimates, approximately 4.4 billion people suffer from $H$. pylori infection globally (5). In recent years, studies of $H$. pylori have become increasingly extensive, and the relationships between $H$. pylori and various extragastric diseases have gradually been reported (6), including pregnancy-related diseases, such as HG, PE, fetal growth restriction (FGR), premature delivery, abortion, and fetal malformation (7-10). Pregnant women are one of the groups most vulnerable to $H$. pylori infection (11), and a meta-analysis by Azami et al. reported the global incidence of $H$. pylori infection in pregnant women to be $46 \%$ (12).

Diabetes in pregnancy (DIP) includes pre-pregnancy diabetes mellitus (PGDM) and gestational diabetes mellitus (GDM). According to statistics from the United States, the incidence of DIP was 7\%, with GDM accounting for $86 \%$ of DIP cases (13). Due to poor control of blood glucose before and during pregnancy, DIP can significantly increase the risk of adverse pregnancy outcomes $(14,15)$, such as miscarriage, stillbirth, premature birth, low birth weight, macrosomia, and neonatal death. Therefore, it poses a serious public health issue around the world. For DIP patients with $H$. pylori infection, it has not yet been established whether having the double hazards of high blood glucose and $H$. pylori infection further promotes the occurrence of pregnancy-related diseases and poor fetal development. It is also unclear whether $H$. pylori screening and eradication therapy is necessary before pregnancy. Therefore, we designed this prospective study to determine the association of $H$. pylori infection with pregnancyrelated diseases and fetal development in women with DIP. We present the following article in accordance with the STROBE reporting checklist (available at http://dx.doi. org/10.21037/atm-21-1209).

\section{Methods}

\section{Study population}

All women who underwent regular prenatal examinations in the perinatal health center of our hospital between June and December 2019 were recruited before reaching 16 weeks of gestation. According to their medical history and the results of a $75 \mathrm{~g}$ oral glucose tolerance test at the $24^{\text {th }}$ week of pregnancy, the participants were divided into a normal control group (NC group), a GDM group, and pre-pregnancy diabetes mellitus group (PGDM group). According to the results of an $H$. pylori serum antibody detection test, each group was further divided into two subgroups: an $H$. pylori positive subgroup (HP+ subgroup) and an $H$. pylori negative group ( $H P$ - subgroup). All participants were given diabetes-related education, guidance on diet and exercise, and regular hypoglycemic therapy. Insulin therapy was given to participants whose blood glucose was unsatisfactory after diet and exercise control. Participants were followed up by telephone once a month until 1 month postpartum.

\section{Ethical approval}

All procedures performed in this study involving human participants were in accordance with the Declaration of Helsinki (as revised in 2013). The study was approved by the Ethics Committee of the Second Affiliated Hospital of Zhengzhou University (No.: 2018047) and informed consent was taken from all the patients.

\section{Inclusion criteria}

All participants were primigravidas aged $\leq 35$ years. PGDM was diagnosed according to the 1999 World Health Organization (WHO) diagnostic criteria for diabetes (16). GDM was diagnosed according to the 2019 diagnostic criteria of the American Diabetes Association (17).

\section{Exclusion criteria}

Women meeting any of the following criteria were 
excluded: (I) non-natural pregnancy; (II) a history of habitual abortion, stillbirth, or induced labor for fetal malformation, or other adverse pregnancy outcomes; (III) twin or multiple pregnancy; (IV) liver, kidney, or cardiac dysfunction; (V) a history of gastrointestinal resection; (VI) a history of smoking; and (VII) a history of family or genetic disease.

\section{Data extraction}

Participant data including age, weight, blood pressure, and the levels of fasting plasma glucose (FPG), glycated hemoglobin (HbA1c), alanine aminotransferase (ALT), aspartate aminotransferase (AST), $\gamma$-glutamyl transpeptidase $(\gamma$-GGT), creatinine $(\mathrm{Cr})$, and urea were collected at recruitment. Blood pressure and urinary protein levels were measured in different periods of pregnancy for the diagnosis of PIH and PE. Participants' weight and urine ketone levels were recorded in different periods of pregnancy for the diagnosis of HG. For the diagnosis of anemia of pregnancy, the level of hemoglobin $(\mathrm{Hb})$ was measured, and the serum ferritin level was further detected when $\mathrm{Hb}<105 \mathrm{~g} / \mathrm{L}$. The fetal developmental status (including the biparietal diameter, head circumference, abdominal circumference, and femur length) were measured in the $2^{\text {nd }}$ trimester and at the end of the pregnancy. The gestational age and the newborn status (including the Apgar score, weight, and body length of the neonate) were also recorded.

\section{Definitions}

Anemia of pregnancy was defined as a hemoglobin level $<110 \mathrm{~g} / \mathrm{L}$ in early or late pregnancy or $<105 \mathrm{~g} / \mathrm{L}$ in mid pregnancy accompanied by a serum ferritin level $<20 \mu \mathrm{g} / \mathrm{L}$ $(18,19)$. HG was defined as continuous nausea and vomiting in early pregnancy with body weight loss of $>5 \%$ compared with the pre-pregnancy weight and a positive reading for ketones in the urine (20), or pregnancy-related nausea or vomiting $>3$ times a day with a body weight loss of $3 \mathrm{~kg}$ or $>5 \%$ compared with the pre-pregnancy weight and a positive reading for ketones in the urine (21). Miscarriage was defined as spontaneous miscarriage or fetal death before 23 weeks. PE was defined as the onset of hypertension (systolic blood pressure $\geq 140 \mathrm{mmHg}$ or diastolic blood pressure $\geq 90 \mathrm{mmHg})$ and proteinuria $(\geq 300 \mathrm{mg} / 24 \mathrm{~h})$ after 20 weeks of gestation. PIH was diagnosed when hypertension appeared after 20 weeks of gestation in the absence of significant proteinuria. Premature delivery was defined as spontaneous onset of labor or preterm premature rupture of membranes before 37 weeks of gestation (22).

\section{Serology}

At recruitment, 2 to $5 \mathrm{~mL}$ of venous blood was collected from participants' elbows after 8 to 12 hours of fasting. The serum was separated by centrifugation immediately after clotting and stored at $-20{ }^{\circ} \mathrm{C}$. An enzyme-linked immunosorbent assay (ELISA) kit was used to detect antibodies against $H$. pylori in each serum sample, including cytotoxic (CagA), vacuolating toxin (VacA), and urease subunit $\mathrm{A}$ and $\mathrm{B}$ antibodies.

The H. pylori test kit chosen was the H. pyloriImmunoblot Kit 2.0 (Shenzhen Blot Biotech Co., Ltd.) and was used as follows. Before application, the kit needed to be balanced at room temperature for 10 to 15 minutes. The whole bottle was diluted to $250 \mathrm{~mL}$ purified water solution and was left to stand. The required imprinted membrane was put into the reaction tank, and $1 \mathrm{~mL}$ of washing solution and $10 \mu \mathrm{L}$ of serum were added. The reaction tank was then placed in $37^{\circ} \mathrm{C}$ incubator for 30 minutes. After that, the liquid in the reaction tank was discarded and the membrane was dried using absorbent paper. Then, $1 \mathrm{~mL}$ of washing solution was added, the membrane was washed for 1 minute, and then the liquid was discarded. This process was repeated 3 times, and then the membrane was dried using absorbent paper. Next, $0.5 \mathrm{~mL}$ of washing solution and $10 \mu \mathrm{L}$ of enzyme-linked reagent were added into the reaction tank. The reaction tank was put into the $37^{\circ} \mathrm{C}$ incubator for 30 minutes. Then, the liquid in the reaction tank was discarded and the membrane was dried using absorbent paper. Next, $1 \mathrm{~mL}$ of washing solution was added, the membrane was washed for 1 minute, and then the liquid was discarded. This process was repeated 3 times, and then the membrane was dried using absorbent paper. Next, $0.5 \mathrm{~mL}$ chromogenic agent was added into the reaction tank and shaken for 5 minutes to dye the membrane. When the color was clear, the liquid in the reaction tank was discarded, and the imprinted membrane was washed 3 times with tap water to terminate the reaction. Finally, the imprinted membrane was taken out and dried using absorbent paper, and the result was compared with the standard tape. Because none of the pregnant women had received $H$. pylori eradication treatment, samples were considered positive when the presence of any 1 of the anti- $H$. pylori IgG antibodies was detected, as suggested by the test manufacturer. 
Table 1 Demographic data of the three groups

\begin{tabular}{|c|c|c|c|c|c|}
\hline Variable & NC group $(n=208)$ & GDM group $(n=86)$ & PGDM group $(n=62)$ & $\mathrm{F}$ & $\mathrm{P}$ \\
\hline Weight (kg) & $53.94 \pm 7.38$ & $53.92 \pm 5.60$ & $52.64 \pm 4.53$ & 1.021 & 0.362 \\
\hline Systolic pressure $(\mathrm{mmHg})$ & $120.94 \pm 12.86$ & $120.42 \pm 16.00$ & $124.16 \pm 18.61$ & 1.187 & 0.307 \\
\hline Diastolic pressure (mmHg) & $78.05 \pm 8.56$ & $78.94 \pm 8.33$ & $79.85 \pm 8.64$ & 0.896 & 0.409 \\
\hline AST (U/L) & $18.77 \pm 4.73$ & $18.21 \pm 4.43$ & $18.85 \pm 5.19$ & 0.447 & 0.640 \\
\hline$\gamma$-GGT (U/L) & $13.40 \pm 6.89$ & $13.33 \pm 6.99$ & $13.94 \pm 6.89$ & 0.160 & 0.853 \\
\hline $\mathrm{Cr}(\mu \mathrm{mol} / \mathrm{L})$ & $47.02 \pm 8.97$ & $46.59 \pm 11.01$ & $46.39 \pm 8.21$ & 0.096 & 0.908 \\
\hline Urea $(\mathrm{mmol} / \mathrm{L})$ & $3.08 \pm 0.81$ & $3.04 \pm 0.71$ & $3.04 \pm 0.75$ & 0.091 & 0.913 \\
\hline HbA1c (\%) & $4.96 \pm 0.26$ & $5.55 \pm 0.33$ & $5.84 \pm 0.62$ & 108.665 & $0.001^{*}$ \\
\hline
\end{tabular}

*, statistically significant difference between the three groups. FPG, fasting plasma glucose; HbA1c, glycated hemoglobin; ALT, alanine aminotransferase; AST, aspartate aminotransferase; $\gamma$-GGT, $\gamma$-glutamyl transpeptidase; $\mathrm{Cr}$, creatinine.

\section{Statistical analysis}

Data were analyzed in SPSS 20.0 (IBM Corp., Armonk, NY, USA). Measurement data were expressed as mean \pm standard error and were analyzed by one-way analysis of variance (ANOVA) or a $t$-test. Count data were expressed as cases or rates and were analyzed using the $\chi^{2}$ test.

\section{Results}

\section{Infection rate of $H$. pylori and demographic data}

A total of 356 pregnant women were recruited before 16 weeks of gestation and were divided into the NC group ( $\mathrm{n}=208$ cases), the GDM group ( $\mathrm{n}=86$ cases), and the PGDM group ( $\mathrm{n}=62$ cases) according to their medical history and the results of a $75 \mathrm{~g}$ oral glucose tolerance test at the $24^{\text {th }}$ week of pregnancy.

The $H$. pylori infection rates in the NC, GDM, and PGDM groups were 48.1\% (100/208), 62.8\% (54/86), and $64.5 \%$ (40/62), respectively. There were no differences in the infection rate of $H$. pylori between the three groups $\left(\chi^{2}=5.987, \mathrm{P}=0.05\right)$.

Next, we further compared the participants by $H$. pylori subtype. The infection rates of type I $H$. pylori in the NC, GDM, and PGDM groups were 24.0\% (50/208), 40.7\% (35/86), and $38.7 \%$ (24/62), respectively. The type I $H$. pylori infection rates in the GDM group and the PGDM group were significantly higher than that in the $\mathrm{NC}$ group $\left(\chi^{2}=6.949, \mathrm{P}=0.031\right)$.

At participant recruitment, the levels of FPG and HbA1c were significantly higher in the GDM and PGDM groups than in the $\mathrm{NC}$ group $(\mathrm{P}<0.05)$. However, no significant differences existed between the three groups with respect to age, weight, blood pressure, ALT, AST, $\gamma$-GGT, Cr, urea, or $\mathrm{Hb}(\mathrm{P}>0.05)$ (Table 1). After hypoglycemic treatment, the FPG and HbA1c levels were similar between the groups $(\mathrm{P}>0.05)$.

According to the results of $H$. pylori antibody detection, each group was further divided into two subgroups: the $H$. pylori positive group ( $H P+$ subgroup) and the $H$. pylori negative group ( $H P-$ subgroup). None of the $H P+$ and $H P$ - subgroups in any of the groups showed significant differences in terms of age, weight, blood pressure, ALT, AST, $\gamma$-GGT, Cr, urea, Hb, FPG, or HbA1c $(\mathrm{P}>0.05)$.

\section{Pregnancy-related diseases}

The incidences of pregnancy-related diseases did not differ significantly between the $\mathrm{NC}-H P+$ subgroup and the NC-HP- subgroup $(\mathrm{P}>0.05)$. However, the GDM-HP+ subgroup had significantly higher incidences of anemia, $\mathrm{PE}$, and premature delivery than the GDM-HP- subgroup 
Table 2 Pregnancy-related diseases in the subgroups

\begin{tabular}{|c|c|c|c|c|c|c|c|c|c|c|c|c|}
\hline $\begin{array}{l}\text { Pregnancy- } \\
\text { related } \\
\text { diseases }\end{array}$ & \multicolumn{4}{|c|}{ NC group } & \multicolumn{4}{|c|}{ GDM group } & \multicolumn{4}{|c|}{ PGDM group } \\
\hline$H G$ & 6 & 2 & 2.416 & 0.120 & 2 & 1 & 0.020 & 0.888 & 4 & 1 & 0.570 & 0.450 \\
\hline $\begin{array}{l}\text { Premature } \\
\text { delivery }\end{array}$ & 14 & 12 & 0.396 & 0.529 & 15 & 3 & 4.112 & $0.043^{*}$ & 20 & 3 & 8.043 & $0.005^{\star}$ \\
\hline
\end{tabular}

${ }^{*}$, statistically significant difference between the $H P+$ subgroup and the $H P$ - subgroup. $\mathrm{HG}$, hyperemesis gravidarum; $\mathrm{PIH}$, pregnancyinduced hypertension; PE, preeclampsia.

Table 3 Pregnancy-related diseases in the three $H P+$ subgroups

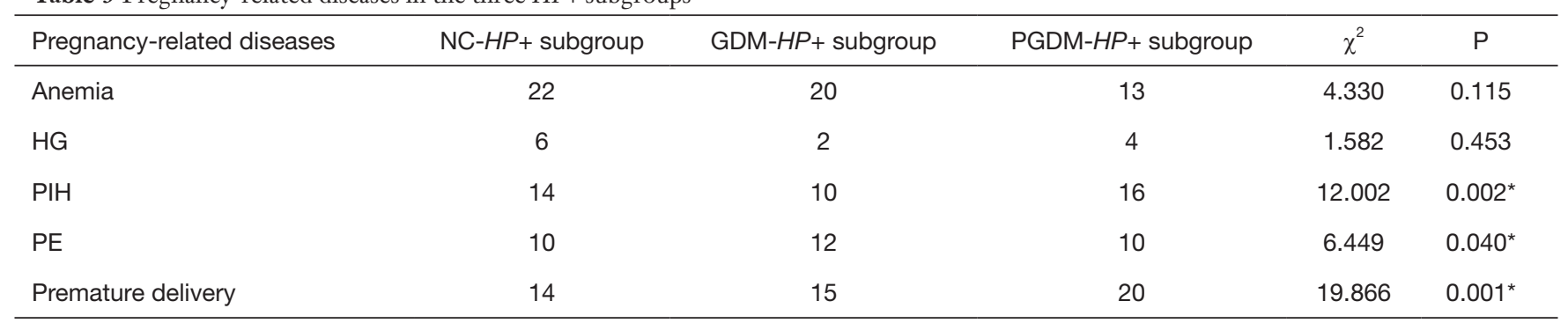

*, statistically significant difference between the three HP+ subgroups. HG, hyperemesis gravidarum; PIH, pregnancy induced hypertension; PE, preeclampsia.

$(\mathrm{P}<0.05)$. Also, the incidences of anemia, $\mathrm{PIH}, \mathrm{PE}$, and premature delivery were significantly higher in the PGDM-HP+ subgroup than in the PGDM-HP- subgroup $(\mathrm{P}<0.05)$ (Table 2).

Next, we further compared the $3 \mathrm{HP}+$ subgroups. The results revealed that the incidences of anemia and HG showed no difference between the $3 \mathrm{HP}+$ subgroups $(\mathrm{P}>0.05)$; however, the incidences of PIH, PE, and premature delivery were significantly higher in the GDM$H P+$ and PGDM-HP+ subgroups than in the NC-HP+ subgroup $(\mathrm{P}<0.05)$ (Table 3).

\section{Fetal development and neonatal conditions}

In the $\mathrm{NC}$ group, none of the indexes showed a significant difference between the $\mathrm{NC}-\mathrm{HP}+$ subgroup and $\mathrm{NC}$ $H P$ - subgroup in the $2^{\text {nd }}$ trimester of pregnancy $(\mathrm{P}>0.05)$. However, at the end of pregnancy, the biparietal diameter, head circumference, abdominal circumference, Apgar score, and neonatal weight were significantly higher in the NC$H P$ - subgroup than in the $\mathrm{NC}-H P+\operatorname{subgroup}(\mathrm{P}<0.05)$, although there were no significant differences in femur length or neonatal body length between the two subgroups ( $>>0.05)$ (Table 4).

In the GDM group, none of the indexes showed a significant difference between the GDM-HP+ subgroup and GDM- $H P$ - subgroup in the $2^{\text {nd }}$ trimester of pregnancy $(\mathrm{P}>0.05)$. However, at the end of pregnancy, all indexes, including biparietal diameter, head circumference, abdominal circumference, femur length, Apgar score, neonatal weight, and body length, were higher in the GDM-HP- subgroup than in the GDM-HP+ subgroup $(\mathrm{P}<0.05)$ (Table 4).

In the PGDM group, the biparietal diameter, head circumference, abdominal circumference, and femur length in the PGDM-HP - subgroup were greater than those in the PGDM-HP+ subgroup, both in the $2^{\text {nd }}$ trimester of pregnancy and at the end of pregnancy $(\mathrm{P}<0.05)$. Furthermore, the neonatal weight, body length, and Apgar score were also higher in the PGDM-HP- subgroup than in the PGDM-HP+ subgroup (Table 4).

One case of fetal death occurred in the NC group, and 


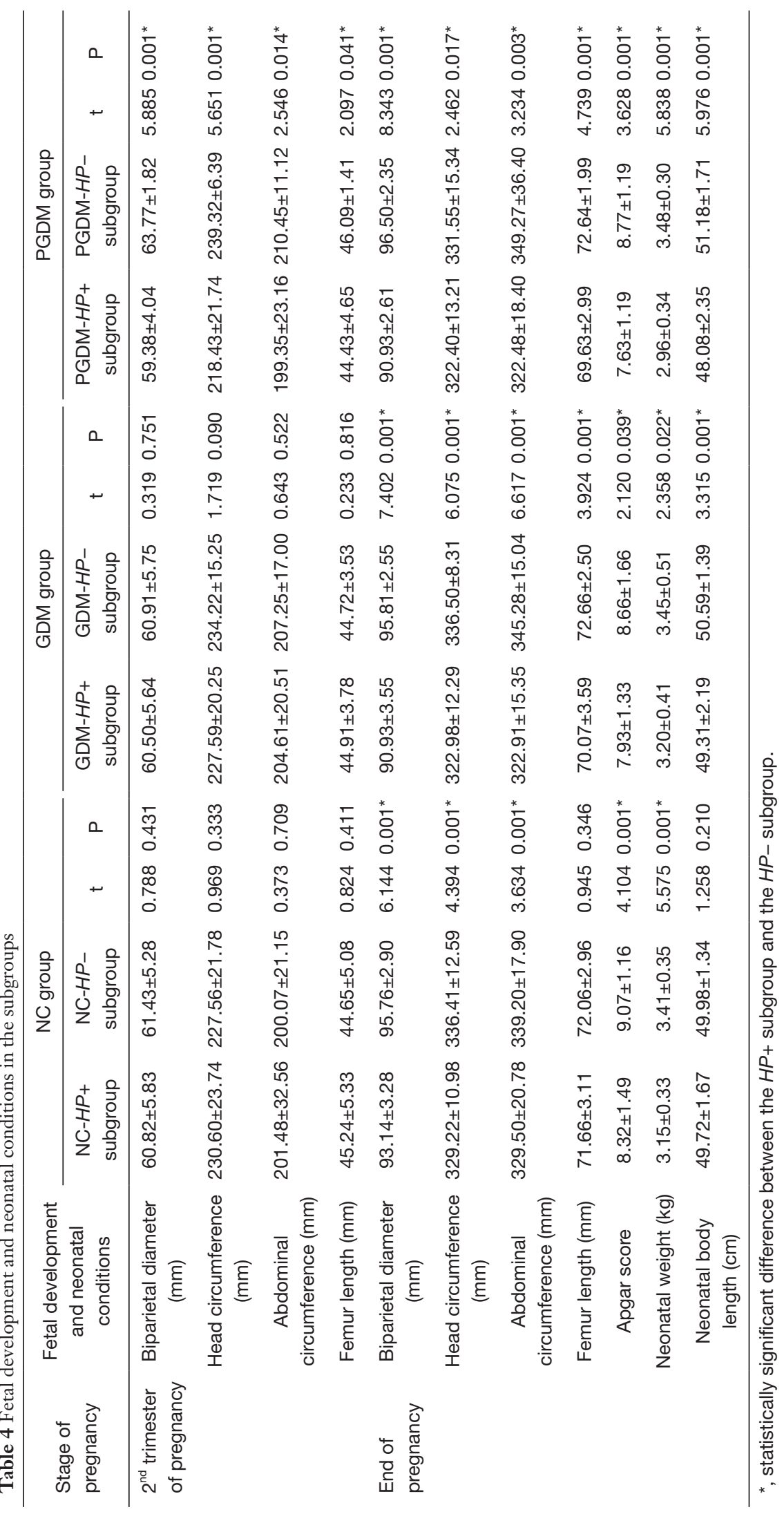


Table 5 Fetal development and neonatal conditions in the three $H P+$ subgroups

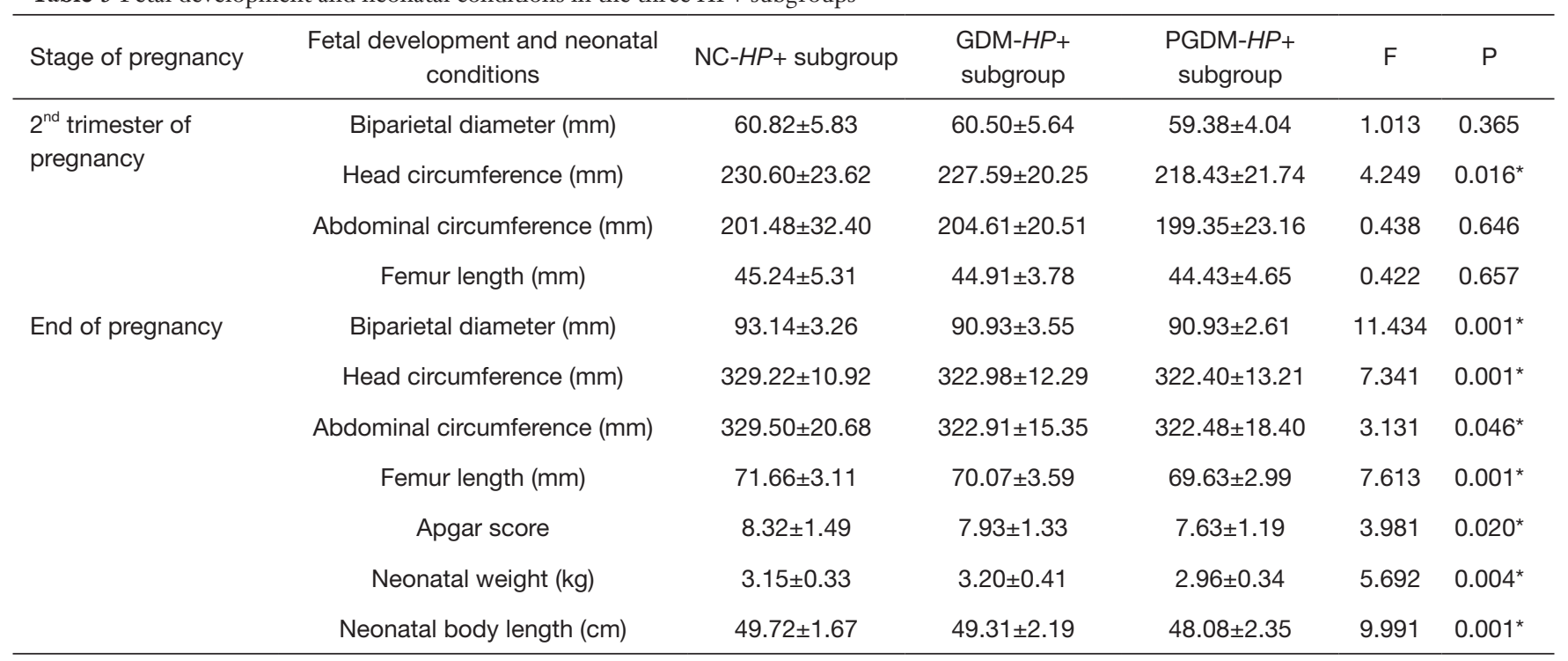

*, statistically significant difference between the three $H P+$ subgroups.

no cases occurred in the GDM and PGDM groups.

Again, we compared the data of the $3 \mathrm{HP}+$ subgroups. The results revealed that only the head circumference showed a significant difference in the $2^{\text {nd }}$ trimester of pregnancy $(\mathrm{P}<0.05)$. At the end of pregnancy, all indexes in the NC-HP+ subgroup, including biparietal diameter, head circumference, abdominal circumference, femur length, Apgar score, neonatal weight, and body length were greater than those in the GDM-HP+ and PGDM-HP+ subgroups $(\mathrm{P}<0.05)$ (Table 5).

\section{Discussion}

To our knowledge, this is the first clinical observational investigation to assess the relationships of $H$. pylori infection and DM with pregnancy-related diseases and fetal development. Our findings indicated that $H$. pylori infection was extremely common in women with DIP, especially those with type I $H$. pylori infection. The influence of $H$. pylori on pregnancy-related diseases and fetal development was further aggravated by DM. In normal pregnant women, $H$. pylori did not show an association with pregnancyrelated diseases; however, it aggravated the incidences of pregnancy-related diseases in women with DIP. The harm caused by $H$. pylori to the fetuses and neonates of women with DIP was also evident, and it could lead to poor fetal and neonatal development.

There are many methods to detect Helicobacter pylori, including serological examination, C13 and C14 breath test, gastric mucosal biopsy and so on. C13 and C14 are radioisotopes and may cause potential health hazards to pregnant women. Gastric mucosal biopsy under gastroscope is invasive examination. Pregnant women will be more worried, which will lead to further reduction of sample size and it is too difficult to implement. Due to the advantages of being simple, inexpensive, and non-invasive, serological detection is the first choice for the diagnosis of $H$. pylori infection during pregnancy. The $\mathrm{IgG}$ antibody may reflect a current infection or prior exposure (23). The sensitivity and specificity of $\operatorname{IgG}$ antibody are reported to be $80-100 \%$ and $69-95 \%$, respectively (24-26). CagA protein and VacA protein are the main markers of $H$. pylori toxicity (27). According to the presence or absence of CagA protein, $H$. pylori infections can be divided into two types: type I is a highly toxic strain with CagA, and type II is a strain without CagA. Infection with type I $H$. pylori can lead to duodenal ulcers, gastric cancer, and extragastric diseases (28). Our study showed that $H$. pylori infection was extremely common in women with DIP. The $H$. pylori infection rate in the GDM group and the PGDM group was $62.8 \%$ and $64.5 \%$, respectively, and the infection rate of type I $H$. pylori specifically was $40.7 \%$ in the GDM group and $38.7 \%$ in the PGDM group; these rates were significantly higher than those in NC group. Therefore, it is suggested that $\mathrm{DM}$ can increase $H$. pylori exposure and the infection rate in pregnancy. The main reasons for this are that $\mathrm{DM}$ 
causes damage to cellular and immune functions, and enhances sensitivity to infection (29). Furthermore, it can weaken gastrointestinal peristalsis and acid secretion, thus promoting the colonization and infection of pathogens in the intestine (30). Due to immune adaptation during pregnancy (31), pregnant women are susceptible to $H$. pylori infection (32). When pregnancy is complicated with DM, the risk of infection further increases.

Results of studies on the relationship between DM and adverse pregnancy outcomes have been relatively consistent $(14,15)$. High blood glucose can cause maternal and fetal complications, such as spontaneous abortion, fetal malformation, preeclampsia, stillbirth, macrosomia, neonatal hypoglycemia, and neonatal hyperbilirubinemia, which can seriously affect the health of the mother and infant. Infection might play a role in the etiology and pathogenesis of pregnancy complications and poor fetal development in DIP. In this study, the high infection rate of type I $H$. pylori in particular may have contributed to the higher incidences of pregnancy-related diseases in the GDM and PGDM groups. We further compared the incidence rates of pregnancy-related diseases and fetal development between the $H P+$ and $H P$ - subgroups in each group. The results showed that $H$. pylori infection could increase the incidences of pregnancy-related diseases and hinder fetal development.

$H$. pylori may participate in the pathogenesis of gestational diseases through a variety of mechanisms. First, H. pylori absorbs essential nutrients from the host, including iron, vitamin B12, and folic acid, which leads to the malabsorption of nutrients in pregnancy, ultimately resulting in severe iron deficiency anemia; gestational anemia is an important factor in fetal development. Second, H. pylori can also lead to systemic endothelial dysfunction by inducing inflammation and oxidative stress, and it participates in the pathogenesis of PE (33). In vitro experiments have shown that these anti-CagA antibodies mainly recognize the antigens located on the surface of endothelial cells. Trophoblast cells have been found to mainly show an endothelial phenotypic profile. The antibodies cross-react with trophoblast cells, reducing the invasive ability of placental trophoblasts $(34,35)$. Therefore, CagA-positive $H$. pylori strains do not only aggravate the inflammatory reaction in the body during pregnancy, but also cause the typical placental abnormalities in PE. Third, several non-genital tract infections, by way of maternal bacteremia and transplacental passage, can cause an intrauterine infection, leading to premature delivery (36-38);
H. pylori infection may induce premature delivery through a similar pathway.

Anemia in pregnancy is an important factor that endangers the health of the mother and infant. It is associated with premature delivery and a low birth weight (39). PE is a type of PIH. Approximately 10\% of women suffer from hypertension during pregnancy, among which $\mathrm{PE}$ accounts for $2 \%$ to $8 \%$ (40). Moreover, approximately $10 \%$ to $15 \%$ of maternal death is directly related to PE (41). Premature delivery accounts for $75 \%$ of perinatal mortality and more than half of long-term morbidity in infants $(42,43)$. Therefore, $H$. pylori infection not only causes pregnancyrelated diseases, but also results in poor fetal development and adverse pregnancy outcomes.

Our results have demonstrated that the double impact of high blood glucose and $H$. pylori infection in women with DIP significantly increases the risks of pregnancy-related diseases and poor fetal development compared to those in normal pregnant women and women with DM or $H$. pylori infection alone.

\section{Conclusions}

In summary, our study has shown that $H$. pylori infection is extremely common in DIP. Infection with $H$. pylori can increase the risks of pregnancy-related diseases and poor fetal development in women with DIP. These risks are higher for women with concurrent $H$. pylori infection and DM than for women with DM or $H$. pylori infection alone. $H$. pylori screening and eradication therapy before pregnancy may aid in preventing pregnancy-related diseases and support fetal development in women with DIP. However, the reliability of our results is controversial due to the many influencing factors in clinical studies and the number of patient samples is too small. Also, we did not further investigate the mechanism underlying the effects of H. pylori infection on outcomes in DIP; our future research will focus on uncovering this mechanism.

\section{Acknowledgments}

Funding: This work was supported by the Medical Science and Technology Research Plan of Henan Province in 2018 (grant No. 2018020146).

\section{Footnote}

Reporting Checklist: The authors have completed the 
STROBE reporting checklist. Available at http://dx.doi. org/10.21037/atm-21-1209

Data Sharing Statement: Available at http://dx.doi. org/10.21037/atm-21-1209

Conflicts of Interest: All authors have completed the ICMJE uniform disclosure form (available at http://dx.doi. org/10.21037/atm-21-1209). The authors have no conflicts of interest to declare.

Ethical Statement: The authors are accountable for all aspects of the work in ensuring that questions related to the accuracy or integrity of any part of the work are appropriately investigated and resolved. All procedures performed in this study involving human participants were in accordance with the Declaration of Helsinki (as revised in 2013). The study was approved by the Ethics Committee of the Second Affiliated Hospital of Zhengzhou University (No.: 2018047) and informed consent was taken from all the patients.

Open Access Statement: This is an Open Access article distributed in accordance with the Creative Commons Attribution-NonCommercial-NoDerivs 4.0 International License (CC BY-NC-ND 4.0), which permits the noncommercial replication and distribution of the article with the strict proviso that no changes or edits are made and the original work is properly cited (including links to both the formal publication through the relevant DOI and the license). See: https://creativecommons.org/licenses/by-nc-nd/4.0/.

\section{References}

1. Marshall BJ, Barrett LJ, Prakash C, et al. Urea protects Helicobacter (Campylobacter) pylori from the bactericidal effect of acid. Gastroenterology 1990;99:697-702.

2. Sgouras DN, Trang TT, Yamaoka Y. Pathogenesis of Helicobacter pylori Infection. Helicobacter 2015;20:8-16.

3. Everhart JE. Recent developments in the epidemiology of Helicobacter pylori. Gastroenterol Clin North Am 2000;29:559-78.

4. Bruce MG, Maaroos HI. Epidemiology of Helicobacter pylori infection. Helicobacter 2008;13:1-6.

5. Hooi JKY, Lai WY, Ng WK, et al. Global Prevalence of Helicobacter pylori Infection: Systematic Review and Meta-Analysis. Gastroenterology 2017;153:420-9.

6. Banić M, Franceschi F, Babić Z, et al. Extragastric manifestations of Helicobacter pylori infection. Helicobacter 2012;17:49-55.

7. Kazerooni T, Taallom M, Ghaderi AA. Helicobacter pylori seropositivity in patients with hyperemesis gravidarum. Int J Gynaecol Obstet 2002;79:217-20.

8. Cardaropoli S, Rolfo A, Piazzese A, et al. Helicobacter pylori's virulence and infection persistence define preeclampsia complicated by fetal growth retardation. World J Gastroenterol 2011;17:5156-65.

9. Eslick GD, Yan P, Xia HH, et al. Foetal intrauterine growth restrictions with Helicobacter pylori infection. Aliment Pharmacol Ther 2002;16:1677-82.

10. Cardaropoli S, Piazzese A, Piccoli E, et al. Is Helicobacter pylori infection a risk factor for miscarriage? Placenta 2013;34:A37-A38.

11. Cardaropoli S, Giuffrida D, Piazzese A, et al. Helicobacter pylori seropositivity and pregnancy-related diseases: a prospective cohort study. J Reprod Immunol 2015;109:41-7.

12. Azami M, Nasirkandy MP, Mansouri A, et al. Global Prevalence of Helicobacter Pylori Infection in Pregnant Women: A Systematic Review and Meta-Analysis Study. Inter J Women Health and Reprod Sci 2017;5:30-6.

13. Correa A, Bardenheier B, Elixhauser A, et al. Trends in prevalence of diabetes among delivery hospitalizations, United States, 1993-2009. Matern Child Health J 2015;19:635-42.

14. Corrado F, Pintaudi B, D'Anna R, et al. Perinatal outcome in a Caucasian population with gestational diabetes and preexisting diabetes first diagnosed in pregnancy. Diabetes Metab 2016;42:122-5.

15. Kulshrestha V, Agarwal N. Maternal complications in pregnancy with diabetes. J Pak Med Assoc 2016;66:S74-S77.

16. Alberti KG, Zimmet PZ. Definition, diagnosis and classification of diabetes mellitus and its complications. Part 1: diagnosis and classification of diabetes mellitus provisional report of a WHO consultation. Diabet Med 1998;15:539-53.

17. American Diabetes Association. Standards of medical care in diabetes-2019. Diabetes Care 2019;42:S1-S2.

18. Pavord S, Myers B, Robinson S, et al. UK guidelines on the management of iron deficiency in pregnancy. Br J Haematol 2012;156:588-600.

19. Breymann C, Bian XM, Blanco-Capito LR, et al. Expert recommendations for the diagnosis and treatment of irondeficiency anemia during pregnancy and the postpartum period in the Asia-Pacific region. J Perinat Med 


\section{Page 10 of 10}

2011;39:113-21.

20. Goodwin TM. Hyperemesis gravidarum. Obstet Gynecol Clin North Am 2008;35:401-17.

21. Golberg D, Szilagyi A, Graves L. Hyperemesis gravidarum and Helicobacter pylori infection: a systematic review. Obstet Gynecol 2007;110:695-703.

22. Murphy DJ. Epidemiology and environmental factors in preterm labour. Best Pract Res Clin Obstet Gynaecol 2007;21:773-89.

23. Feldman M, Cryer B, Lee E, et al. Role of seroconversion in confirming cure of Helicobacter pylori infection. JAMA 1998;280:363-5.

24. Cutler AF, Havstad S, Ma CK, et al. Accuracy of invasive and noninvasive tests to diagnose Helicobacter pylori infection. Gastroenterology 1995;109:136-41.

25. Logan RP, Walker MM. ABC of the upper gastrointestinal tract: Epidemiology and diagnosis of Helicobacter pylori infection. BMJ 2001;323:920-2.

26. Lerang F, Moum B, Mowinckel P, et al. Accuracy of seven different tests for the diagnosis of Helicobacter pylori infection and the impact of $\mathrm{H} 2$-receptor antagonists on test results. Scand J Gastroenterol 1998;33:364-9.

27. Blaser MJ, Atherton JC. Helicobacter pylori persistence: biology and disease. J Clin Invest 2004;113:321-33.

28. Graham DY, Yamaoka Y. H. pylori and cagA: relationships with gastric cancer, duodenal ulcer, and reflux esophagitis and its complications. Helicobacter 1998;3:145-51.

29. Koh GC, Peacock SJ, van der Poll T, et al. The impact of diabetes on the pathogenesis of sepsis. Eur J Clin Microbiol Infect Dis 2012;31:379-88.

30. De Block CE, De Leeuw IH, Pelckmans PA, et al. Current concepts in gastric motility in diabetes mellitus. Curr Diabetes Rev 2006;2:113-30.

31. Chang J, Streitman D. Physiologic adaptations to pregnancy. Neurol Clin 2012;30:781-9.

32. Lanciers S, Despinasse B, Mehta DI, et al. Increased susceptibility to Helicobacter pylori infection in pregnancy. Infect Dis Obstet Gynecol 1999;7:195-8.

Cite this article as: Li J, Fan M, Ma F, Zhang S, Li Q. The effects of Helicobacter pylori infection on pregnancy-related diseases and fetal development in diabetes in pregnancy. Ann Transl Med 2021;9(8):686. doi: 10.21037/atm-21-1209

\section{Li et al. Association of $\boldsymbol{H}$. pylori infection on diabetes in pregnancy}

33. UstUn Y, Engin-UstUn Y, Ozkaplan E, et al. Association of Helicobacter pylori infection with systemic inflammation in preeclampsia. J Matern Fetal Neonatal Med 2010;23:311-4.

34. Franceschi F, Di Simone N, D'Ippolito S, et al. Antibodies anti-CagA cross-react with trophoblast cells: a risk factor for pre-eclampsia? Helicobacter 2012;17:426-34.

35. Franceschi F, Niccoli G, Ferrante G, et al. CagA antigen of Helicobacter pylori and coronary instability: insight from a clinico-pathological study and a meta-analysis of 4241 cases. Atherosclerosis 2009;202:535-42.

36. Goldenberg RL, Culhane JF, Johnson DC. Maternal infection and adverse fetal and neonatal outcomes. Clin Perinatol 2005;32:523-59.

37. Romero R, Oyarzun E, Mazor M, et al. Meta-analysis of the relationship between asymptomatic bacteriuria and preterm delivery/low birth weight. Obstet Gynecol 1989;73:576-82.

38. Offenbacher S, Jared HL, O'Reilly PG, et al. Potential pathogenic mechanisms of periodontitis associated pregnancy complications. Ann Periodontol 1998;3:233-50.

39. Singla PN, Tyagi M, Kumar A, et al. Fetal growth in maternal anemia. J Trop Pediatr 1997;43:89-92.

40. Uzan J, Carbonnel M, Piconne O, et al. Pre-eclampsia: pathophysiology, diagnosis, and management. Vasc Health Risk Manag 2011;7:467-74.

41. Suman P, Gandhi S, Kumar P, et al. Prospects of electrochemical immunosensors for early diagnosis of preeclampsia. Am J Reprod Immunol 2017. doi: 10.1111/ aji.12584.

42. McCormick, MC. The contribution of low birth weight to infant mortality and childhood morbidity. N Engl J Med 1985;312:82-90.

43. Saigal S, Doyle LW. An overview of mortality and sequelae of preterm birth from infancy to adulthood. Lancet 2008;371:261-9.

(English Language Editor: J. Reynolds) 